\title{
A Blueprint for Pervasive Self-Care Infrastructures
}

\author{
George Roussos \\ Birkbeck College, University of London \\ g.roussos@bbk.ac.uk
}

\begin{abstract}
Self Care is a recent initiative by the Department of Health in the UK that aims to treat patients with longterm conditions sooner, nearer to home and earlier in the course of their disease. To this end, it places the point-of-care in the community and often at the patients' own home. In this paper we discuss the building blocks of Pervasive Self Care, a ubiquitous computing service infrastructure that can play a critical role in supporting patients with long tem conditions to manage their case. This service employs computing elements embedded in the home environment and the body so as to provide contextaware case management. The proposed architecture brings together islands of pervasive computing functionality into an integrated end-to-end architecture. In doing so, it aims to introduce a generic reference model for the delivery of pervasive self-care services that can help limit the considerable amount of duplication in current work in this field.
\end{abstract}

\section{Introduction}

According to the Department of Health, in the UK more than seventeen and a half million people report living with a long term condition (such as diabetes, asthma, adult congenital heart disease or arthritis), which in many cases limits their ability to cope with day-to-day activities. Care for this group, especially the elderly and those with more than one condition, has traditionally been reactive, unplanned and episodic. The effect of this situation is considerably higher load for secondary care services. It is estimated that a mere $5 \%$ of inpatients, many with a long term condition, account for $42 \%$ of all acute bed days, a fact not unrelated to the finding that only about $50 \%$ of medicines are taken as prescribed.

Moreover according to United Nations estimates, by the year 2020 more than $25 \%$ of the UK population will be over 60 years old. This is a substantial increase compared to the same statistics as of 1999, where only $20 \%$ of the population was in this age group. Also, more than $40 \%$ of the elderly (i.e. those over 65 ) enter a nursing home for at least one year, which is a great

\author{
Andy Marsh \\ VMW Solutions Ltd \\ a.marsh@vmwsolutions.com
}

cause of anxiety both to themselves and their families and carers.

To improve this situation a major initiative is underway in the UK aiming to embed an effective, systematic approach to the care and management of patients with a long term condition into local healthand social-care communities. A core ingredient of this strategy is the so-called supported Self Care that is, to collaboratively help individuals and their carers to develop the knowledge, skills and confidence to effectively manage their condition themselves.

In this paper, we report on our current work on a conceptual framework for the development of pervasive Self Care services as a core component in case management for patients with long-term conditions. We propose this model due to the fact that in several pervasive healthcare research projects $[7,11$, $14,16]$ that we are involved in, we have duplicated systems and functionalities as a result of the lack of a common service delivery substrate. Our early experience with a shared foundation for the development of components, services and applications appears to point to the fact that considerable resource savings can be achieved by following this approach, and for this reason, we propose it as a generic model that can be of use to others working in this area. In fact, in section three, we discuss a number of research projects also plagued by this duplication of effort that concerns the infrastructural rather than the innovative aspects of the conducted research. In this paper, we concentrate on the overall systems architecture of such a service and argue why it represents an appropriate blueprint for this task. Wherever appropriate, we discuss our current implementation of a particular subsystem or service.

The paper is organized as follows: in section two we argue that SOA is the appropriate conceptualization for the Pervasive Self Care. Then, we report on related work in pervasive healthcare with a view to identifying common concerns and requirements. In section four, we introduce the overall systems architecture and in sections five to seven we consider in turn each of its components. Finally, we discuss the long-term objectives of our work and its implications. 


\section{Background and Rationale}

Integrated healthcare information systems are highly decentralized and heterogeneous, geographically distributed and often have global scope. As such, they must scale to support large numbers of users; they must be highly reliable to so as to address the distinct and measurable impact of possible failure; and must be trustworthy and thus protect personal information.

Heterogeneity and distribution in particular, imply considerable challenges regarding synchronization and concurrency as well as compatibility. To address these issues numerous platforms and frameworks have been developed around middleware services. Recently, service-oriented architectures (SOA) have emerged as a model that better addresses these requirements. SOA provides a simple and relatively transparent end-to-end solution using the concept of the so-called Enterprise Service Bus that is, a shared communications medium on which services may connect to and use in a plugand-play manner.

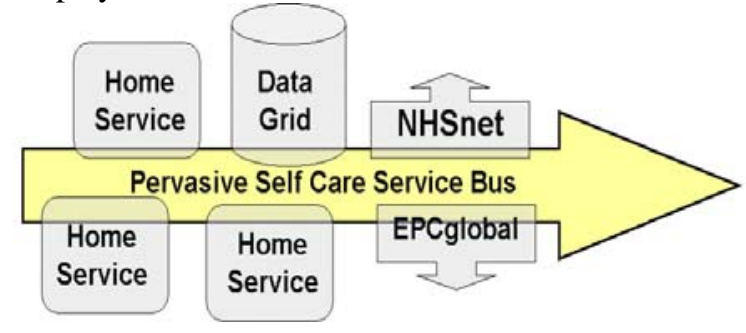

Figure 1. Pervasive Self Care as a Service Oriented Architecture.

Moreover, pervasive technologies for healthcare provision are also gaining prominence. Several (often multidisciplinary) groups are investigating specific solutions in different settings, each having to address efficient and user friendly data gathering, information management, protocols and data specifications, behavioral pattern mining, cognitive assistance, context awareness, proactive notification and many more. The complexity of such tasks leads to often incompatible assumptions about the operational environment of pervasive healthcare. It is not uncommon that targeted systems developed to address a specific concern are in practice isolated islands of functionality that assume that an often closed or incomplete system is in place, for example they expect electronic health record (EHR) information to be available is a specific, proprietary format. Moreover, such systems are often tied to a particular technical solution and extending their functionality to associated devices or platforms is next to impossible.

Yet, it is possible to work within a unified reference model based on SOA that allows systems to be developed with a view of operating alongside other services and sharing a public pervasive computing infrastructure in a Lego-like manner (cf. Figure 1). In this paper we propose such a framework developed across several pervasive healthcare projects We also introduce the data standards and protocols employed to glue together the different components into a common, public pervasive Self Care service.

\section{Related Work}

Despite increased interest in pervasive healthcare most attempts have focused on specific aspects of service provision, isolated from the real-world situation of currently operational systems. In this section we briefly discuss several such services.

The Georgia Tech Aware Home [1] and the Smart Medical Home [12] at University of Rochester examine the role of domestic technologies in providing a supportive environment for the elderly. Such agingin-place projects focus primarily on monitoring and notification and often combine a personal information manager and a model of daily activities developed using a combination of sensor signals collected via a home network. This approach is also employed by MIT's Changing Places [5], which provides a contextaware proactive health communications application using wearable and environmental sensing.

Context-awareness is also the main ingredient for two projects which aim to develop proactive memory aids namely, Assisted Cognition at the University of Washington and Autominder [13] at Michigan and Carnegie Mellon Universities. One application of the later is the Adaptive Prompter, which employs a sensor network to record activities in the home, analyzes the data using artificial intelligence techniques, and supports decision-making, intervention and assistance in everyday activities. Research on mechanism that can help reduce the constraints of cognitive impairment using biosensors is carried out at the OHSU's Point-ofCare Engineering Laboratory [6].

Furthermore, the Center for Pervasive Healthcare at the University of Aarhus explores pervasive computing for use by consultants in the hospital for example, via the so-called context-aware hospital bed and collaborative radiology [2]. In the UK, two research initiatives supported by the DTI Nextwave program aim to develop effective health and wellness monitoring sensors namely, "Care in the Community" and "Ubiquitous Computing for Healthcare in the Community" projects. Both currently focus on the development of devices capable to collect and record personal vital signs and environmental conditions. 
At least three technology startups currently offer wireless real-time remote heart monitoring systems. Cardionet uses a mobile telephony enabled PDA connected wirelessly to an electro-cardiograph (ECG) to record and transmit data to a remote diagnostic server for further processing. Medtronic uses an implanted defibrillator to collect data onto an attached wearable device, which later connects to the fixed telephony network. Finally, Biotronic offers the more ambitious approach where an implanted cardioverterdefibrillator proactively communicates with a customized mobile telephone when it detects a problem; with the information relayed to a centralized sever for analysis. Each company uses proprietary, closed and incompatible protocols to encode and transmit the data to the diagnostic systems. VMW Solutions offers a general-purpose wearable wireless router that acts as a local data router for a variety of devices with optional local or cellular wireless network connectivity.

Finally, medication compliance using radio frequency identifiers (RFID) has also attracted considerable interest. For example, current work on EPCglobal has developed an overlay network that can be used to map Electronic Product Codes --- stored on the RFID embedded in the medicine packaging --- to specific suppliers and product data over the Object Naming and EPC Information Services. It can thus be used to track medications from manufacture to consumption. Although the current focus in this area is on anti-counterfeiting, the availability of RFID offers a clear opportunity for healthcare applications in the spirit of Accenture Labs' medicine cabinet [17].

\section{Systems Architecture}

One of the main tasks in developing the Pervasive Self Care SOA specification is to provide a common mechanism and associated semantics for the exchange of messages. We have defined the semantics of messaging by extending the Virtual Medical Worlds Protocol (VMWP) to use web services as a carrier. VMWP [11] has been used for several years in the context of telecare and 3G-mobile healthcare services and it has been developed to provide for a fairly complete set of remote sensing and notification situations. In the recent past, the protocol has been modified for use over different protocols including Short Messaging Service and GPRS. In the case of Pervasive Self Care the main extension relates to the development of appropriate XML envelopes for the existing protocol structures. Messages are then relayed via a gateway service acting a content router.
The final component of the messaging system required by the Pervasive Self Care service bus is a mediator for retrieving data from the EPCglobal network. Messages to the ONS and EPC IS are encoded appropriately [4] and relayed.

A network-centric view of Pervasive Self Care is offered by the Muti-Sphere Reference Model recently introduced by the Wireless World Research Forum [3]. Figure 2 shows an adaptation of this model as it applies to the Pervasive Self Care service: level one is the body area network supported by a wearable router which interacts with various body sensors and also acts as the gateway to the next level sphere, the home network. The home sphere integrates three different networks, namely the body area network, the medical cabinet (RFID tracking) and the environmental sensing network. The home server can interface as appropriate to these sub-systems, fuse the collected data and relay to the next layer. The global Self Care sphere is a data Grid service that receives data from all participating home servers. Data on the Grid is post-processed and associated with related computational automatic diagnosis mechanisms that track generated data and proactively monitor specific conditions. These mechanisms initiate responses (often notifications) in response to identified critical conditions.

\section{Body Sphere Service}

At the core of the system is the body area network (BAN), which employs an adaptable, wearable gateway device to harvest data from a variety of holters, wearables and biosensors. The device can either initiate communication with sensors and request data or receive notification of an incoming data stream. In our current implementation, we are using the VMW Solutions i-button device which also supports biometric authentication at very low cost (below $£ 3$ at this time).

Sensor data is encoded in an appropriate XMLbased format, and stored locally on the gateway until it can connect to an appropriate server and transmit the information for further processing. This approach allows for more intelligence located closer to the primary medical sensing devices, which results in reduced power requirements due to aggregation of readings and thus lower communication needs. It also provides for:

- increased interoperability with sensing devices; the gateway provides discovery and selfconfiguration mechanisms based on a local database of known device profiles that maintains appropriate data exchange specifications, 
- increased robustness; the gateway operates also as a local cache for harvested data, and,
- better security; the gateway implements access control mechanisms for collected data notably by employing fingerprint authentication for the user.

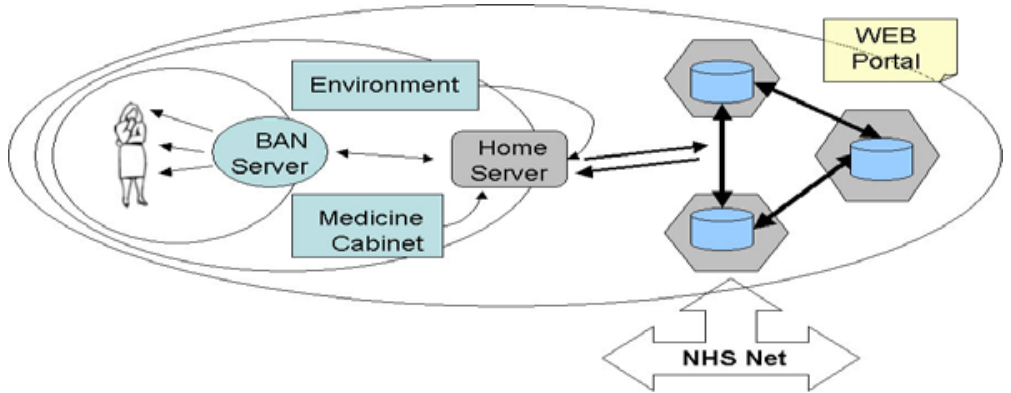

Figure 2. Pervasive Self Care as a Multi-sphere Reference Model.

Currently we encode collected information using the intelligent note or i-note specification (cf. Figure 3). This approach allows for i-notes to be associated with particular applications and also viewed after different stylesheet transformations depending on the role and the authority level of the personal accessing the data for example whether this is a doctor, a patient or a carer. Several notes may be grouped together in an i-pad and used for particular diagnostic tasks.

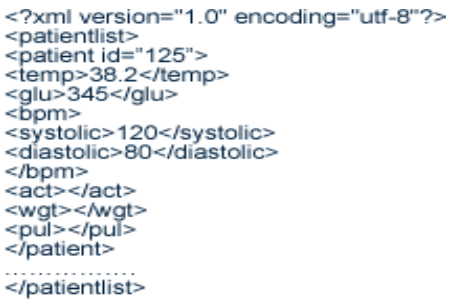

Figure 3. An i-pad example.

\section{Home Sphere}

The home sphere has a dual role: it supports the body area network and the home environment in collecting and pre-processing sensed data while at the same time operates as a media delivery center thus providing feedback to the user (cf. Fingure 5). The home gateway uplink can connect wirelessly to the BAN gateway to retrieve harvested data; it can collect environmental data (e.g. temperature, air quality, pathogens and so forth) and medication usage data. This data are cached locally with the home server acting as the intermediary to self-care service sphere. The home gateway downlink receives event notifications from the data grid service (for example medication reminders) and also associated content related to the current situation of the patient (for example weather and pollutant prediction) and can connect to display device (notably the TV set) to display this information.
Currently, we employ an Open Service Gateway Initiative system developed on top of an embedded Linux platform, which communicates with the i-button via Bluetooth. The connection to external networks is provided by ADSL as GPRS speeds are far below the requirement for timely data transfer. We are using a variety of sensor boards for the collection and post processing of environmental conditions, notably the moteiv and Sensor Cube platforms. Communication with EPCglobal overlay services is via a homegrown open source implementation of the ONS and EPC IS specifications.

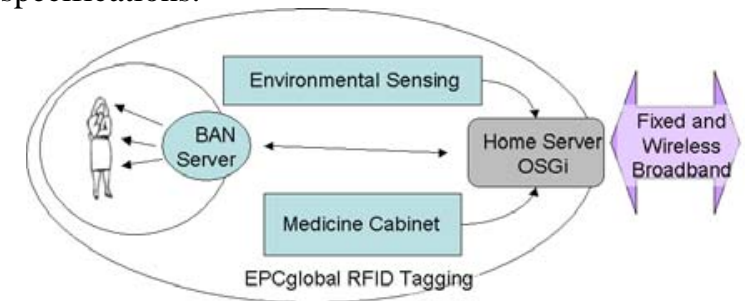

Figure 5. The Home Sphere with gateway services to the Body Sphere and the Self Care data Grid.

\section{Self Care Service Sphere}

The Self Care service sphere acts as the data processing substrate for the system. Data harvested by the system is relayed, stored for processing and related to particular conditions. Data sharing is a critical component and it is supported via a data-grid repository. On these repositories we enable active behavior using Event-Condition-Action rule processors, which define conditions and reactions such as disease detection. At this level security policies are defined and implemented via mapping to lower sphere components, with organization relationships and control exercised. Currently, we are using the Oracle data Grid engine and the new Sensor Based Computing engine in version $10 \mathrm{gR} 2$, to collect, store and process the data. Furthermore, we investigate our own Asene 
language for ECA rule specification to further improve in-network performance of the system.

\section{Long Term Case Studies}

To benchmark our work our long-term aim is to implement two case studies that require a fully developed system. Although currently we are a long way away from this target, by setting specific targets we believe that we can better concentrate our efforts as appropriate.

Disease Detection. In this case we aim to employ a microphysiometer-based biosensor system (measuring $\mathrm{pH}, \mathrm{pO}_{2}$ and $\mathrm{pCO}_{2}$ ) to monitor cell metabolism and thus detect the presence of a particular metabolite or a combination of metabolites. The observed signature can then be matched against a database of known patterns that indicate particular diseases thus providing accurate diagnosis. Although this is possible today using nuclear magnetic resonance spectroscopy, carrying out the diagnostic procedure requires the individual visiting a clinic that holds this massive equipment. Alternatively, a biosensing component [8] combined with an array of silicon based chemical sensors ( $\mathrm{pH}$ ISFET, Severinghaus type $\mathrm{pCO}_{2}$ ISFET and an amperometric $\mathrm{pO}_{2}$-sensor) that communicate with an implantable wireless sensor node can carry out such disease detection procedure at any situation. Indeed, this case study focuses on two main challenges: successful long-term operation of biosensing networks and end-to-end quality of service of the pervasive Self Care platform with hard response times.

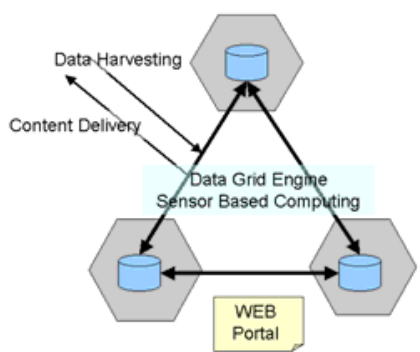

Figure 6.The Self Care Service Data Grid service.

Automatic Artificial Respiration. A variety of diseases attack the respiratory system and make individuals unable to breath on their own, which is also the case in accidents and other anaphylactic situations. In such cases it is necessary to carry out an artificial respiration procedure to support the patient for the duration of the attack. Currently, artificial respiration is only possible in cases where trained personnel and specialized equipment are available and the process is initiated and carried out by a person. We design for a situation where a monolithic pressure biosensor combined with a micro-electromechanical sensor node will be used to initiate artificial respiration unsupervised. This case study poses the challenge of using additional information recorded from environmental sensors and fusing into a global model but also of appropriate expressive languages that can capture effectively and efficiently the situation.

\section{Further Work and Challenges}

Pervasive Self Care systems operate unsupervised to harvest unique patient data. Despite the advantages of this mode of operation, there are also several challenges that must be addressed.

\section{Data and Programming Model}

Pervasive Self Care application programming has unique requirements, which are not adequately catered for by current models for embedded systems development. Two alternative paradigms appear to be appropriate in some cases: the first is based on the well-established model of Query Processing (QP) for sensor databases; and the second is based on the Event-Condition-Action (ECA) rule model. In the first case, we need to explore appropriate data models for medical applications, since current sensor network QPs are limited to a single data space and do not meet the requirements of the case study applications. In the case of the ECA model, we need to further explore the mechanisms for the specification and mining of biosensed data events and patterns with particular emphasis on power efficiency in particular to nondecomposable condition statements.

\section{In-network Storage and Data Reduction}

One of the major challenges for Pervasive Self Care is increasing the longevity of sensor and actuator networks. Software mechanisms have a critical role to play in helping overcome this limitation; in particular in-network data reduction techniques can offer significant benefits. Pervasive Self Care requires that data aggregators be developed with emphasis on error correction and recovery. Unlikely other sensor network systems where errors may be tolerated, in Pervasive Self Care errors may have direct consequences for the well being of the individuals involved and thus error detection is critical in the success of any such system.

\section{Robustness}

Power failures often lead to body and home area network segmentation and network redeployment frequently leads to topology changes. Pervasive Self Care must take into account these changes in network situation so as to develop architectures that are robust in these demanding circumstances. In particular, it is 
necessary to examine the effect on network segment loss on the quality of data harvesting and will examine in-network and external mechanisms for compensation of lost system functionality.

\section{Low-Power Content-based Routing}

The current generation of query processors that are candidates for use in Pervasive Self Care systems mostly use tree-based routing mechanisms that often do not meet adequately the needs of medical applications, which are often characterized by small, incremental updates rather than a single data collection step. Thus, alternative power aware routing techniques should be developed. Pervasive Self Care must develop such routing mechanisms with particular focus on efficient response to the incremental data transfers observed in these applications.

\section{Standardization}

Although in our own work we can choose to employ particular data formats and protocols, for the proposed service architecture to be successful it is necessary that these specifications become commonly available and supported by a multitude of systems.

\section{Conclusions}

In this paper, we present a blueprint for Pervasive Self Care. We assert that such a design is required because current consensus poses significant limitations on experimentation with pervasive computing in healthcare. By introducing this design with its modular components we hope that the duplication of effort currently required to develop infrastructural elements to support specific applications will be removed.

\section{References}

[1] G. Abowd, I. Bobick, E. Essa, E. Mynatt, and W. Rogers, Proceedings of AAAI Workshop and Automation as a Care Giver, Alberta, Canada, July 2002.

[2] J. E. Bardram, "Applications of Context Aware Computing in Hospital Work - Examples and Design Principles”, Proc. ACM SAC 04, Nicosia, Cyprus, March 1417, 2004, pp. $1574-1579$.

[3] K. Crisler, T. Turner, A. Aftelak, M. Visciola, A. Steinhage, M. Anneroth, M. Rantzer, B. von Niman, A. Sasse, M. Tscheligi, S. Kalliokulju, E. Dainesi and A. Zucchella, "Considering the User in the Wireless World", IEEE Comm. Magazine, Vol. 42, No. 9, 2004, pp. 56-62.

[4] C. Floerkemeier, D. Anarkat, T. Osinski and M. Harrison, PML Core Specification 1.0, Auto-ID Center Recommendation, 2003.
[5] S.S. Intille, J. Rondoni, C. Kukla, I. Anacona, and L. Bao. A Context-Aware Experience Sampling Tool. Proceedings of the Conference on Human Factors and Computing Systems, 2003.

[6] H.B. Jimison, M. Pavel, J., Pavel, “Adaptive Interfaces for Home Health", 2nd International Workshop on Ubiquitous Computing for Pervasive Healthcare Applications, October 12-15, Seattle, WA., 2003.

[7] G. E. Karagiannis, V.G. Stamatopoulos, G. Roussos, T. Kotis, M.A. Gatzoulis. "Citizen-centred Health and Lifestyle Management via Interactive TV in patients with Severe Chronic Cardiovascular Diseases: Patient views on the PANACEIA-iTV Health System”. J. Telemed. Telecare, 2005.

[8] E. Lauwers, J. Suls, G. Van der Plas, E. Peeters, W. Gumbrecht, D. Maes, F. Van Steenkiste, G. Gielen and W. Sansen, "A CMOS multi-parameter biochemical microsensor with temperature control and signal interfacing”. Proc. 2001 IEEE In. Solid-State Circuits Conf., 2001, pp. 244-245.

[9] L. Liao, D. Fox, and H. Kautz, "Learning and Inferring Transportation Routines”, Nineteenth National Conference on Artificial Intelligence, San Jose, CA, 2004.

[10] K.J. Liszka, M.A. Mackin, M.J. Lichter, D.W. York, D. Pillai and D.S. Rosenbaum, "Keeping a Beat on the Heart", IEEE Perv. Comp., Vol. 3, No. 4, O 2004, pp. 42-49.

[11] A. Marsh, L. Grandinetti and T. Kauranne, Advanced Infrastructures for Future Healthcare, IOS Press, 2000.

[12] M. Perillo, W. and Heinzelman, "Providing Application QoS Through Intelligent Sensor Management," First IEEE International Workshop on Sensor Network Protocols and Applications (SNPA), 2003.

[13] M. E. Pollack, "Intelligent Technology for an Aging Population: The Use of AI to Assist Elders with Cognitive Impairment," AI Magazine, to appear, 2005.

[14] A. Prentza, S. Maglavera, G. Stalidis, E. Sakka1, I. Lekka, P. Angelidis, L. Leondaridis, N. Maglaveras, D. Koutsouris, "Cost-Effective Health Services for Interactive Lifestyle Management: The PAVACEIA-iTV and the e-Vital Concepts”, Journal of Telecommunications and Information Technology, to appear, 2005.

[15] P.E. Ross, "Managing care through the air", IEEE Spectrum, Vol 41, No. 12, 2004, pp. 26- 31.

[16] Y. Vogiazou, A. Marsh and G. Roussos, "Healthcare Compunetics: An End-to-End Architecture for Self-Care Service Provision", Proc. 1st Int. Work. Body Sensor Networks, 6-7 April 2004, London UK, 2004.

[17] D. Wan. "Magic Medicine Cabinet: A Situated Portal for Healthcare.” Proc. Int. Symp. Handheld and Ubiquitous Computing. September 27--29. Karlsruhe, Germany. 1999. 